\title{
Quantitative Analysis Carbon-Carbon Bonds in a Multiwall Carbon Nanotube using Electron Diffraction
}

Jiong Zhang and J.M. Zuo

\begin{abstract}
Dept. of Materials Science and Engineering and Materials Research Laboratory, University of Illinois at Urbana-Champaign, Urbana, IL 61801, USA
\end{abstract}

Carbon is an important material that has attracted considerable attentions in the past and present. Carbon is also interesting because it can form several types of bonds from single to triple bonds. The Carbon-Carbon $(C-C)$ bond distance changes from the pure single bond of $1.504 \AA$ to pure double bond of $1.334 \AA$ [1]. In carbon nanotubes, $C$ - $C$ bonds are subjected to a large deformation. Study of $\mathrm{C}$ bonds in nanotubes or other nanostructures is in general very difficult. Electron diffraction has been used for structure determination of single and double wall CNTs. However, accurate measurement of $C-C$ bond distances in these cases is difficult from the lack of calibration of camera length and the inclination of nanotubes against the electron beam [2]. Here we report a general electron diffraction procedure for study of $C$ - $C$ bonds in MWCNTs. The analysis method is based on the layer line fitting method reported in [3].

The MWCNTs studied here were synthesized by chemical vapor deposition (CVD) obtained from NANOCYL Company (France). Electron diffraction patterns were recorded from individual MWCNTs in the NED mode using the JEOL 2010F transmission electron microscope (TEM) with a field emission gun at $200 \mathrm{kV}$ installed at University of Illinois. We used a nearly parallel electron nanobeam of about $50 \mathrm{~nm}$ in diameter in NED, and the pattern was recorded on imaging plates (IP) as shown in Figure 1. The diffraction pattern was analyzed first to assign chiral angles and diffraction layer lines to each wall. The layer lines were then fitted to measure the tube diameters, and this fitting takes account of the tube inclination, and the accuracy to determine the diameter has been largely enhanced by this fitting. The deviation could be further reduced by averaging the value from different layer lines of both the first and second order graphene reflections, and from different diffraction patterns taken from the same tube. The layer line positions together with the tube inclination angle were used to determine the helical repeat along the tube direction.

Using the above method, we studied a small diameter MWCNTs of five walls with diameter ranging from $\sim 16$ to $\sim 46 \AA$. The results revealed significant difference between the measured diameter and calculated diameter using the idea graphene $C$ - $C$ bond length of $1.421 \AA$. The smallest innermost wall of $\sim 16 \AA$ in diameter indicates a $3.8 \pm 0.4 \%$ of bond stretching around the tube. This radial stretching was also observed for larger diameter walls, but the amount of stretching reduces as the tube diameter increases. For the outmost wall with $\sim 46 \AA$ diameter, there is still $0.6 \pm 0.2 \%$ stretching as shown in Fig.2. It's obvious this radial stretching has strong diameter dependence, which shows larger stretching with smaller diameter and higher curvature. However, the axial periodicity along the tube direction is the same as the idea CNT value, and no diameter dependence was observed. This could be explained by an anisotropic version of the graphene structure model, called quinoid and discussed long time ago by Pauling [4]. (The work is supported by DOE BES grant DOE DEFG02-01ER45923 and NSF of China 60728102.) 


\section{References}

[1] Pauling. The nature of the chemical bond. Corneel University Press. New York, 1960.

[2] L. C. Qin, Reports on Progress in Physics, 69 (2006) 2761.

[3] J. M. Zuo, T. Kim, A. Celik-Aktas, et al., Zeitschrift Fur Kristallographie, 222 (2007) 625.

[4] L. Pauling, Chemistry, 56 (11966) 1646.
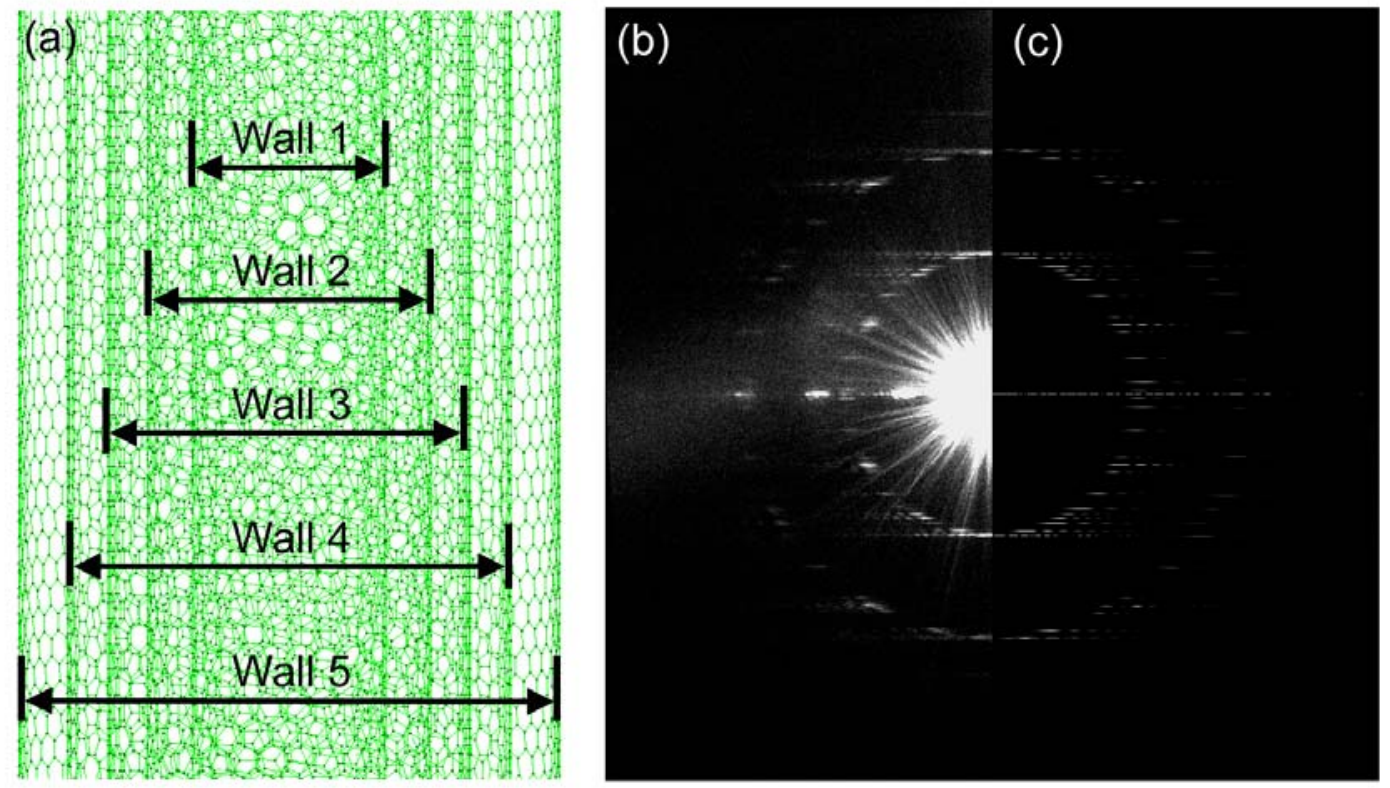

FIG. 1. The structure model (a) is constructed based on the analysis of the diffraction pattern (b). The relative layer lines positions match well of the experimental ED (b) and the simulated ED (c).

(a)

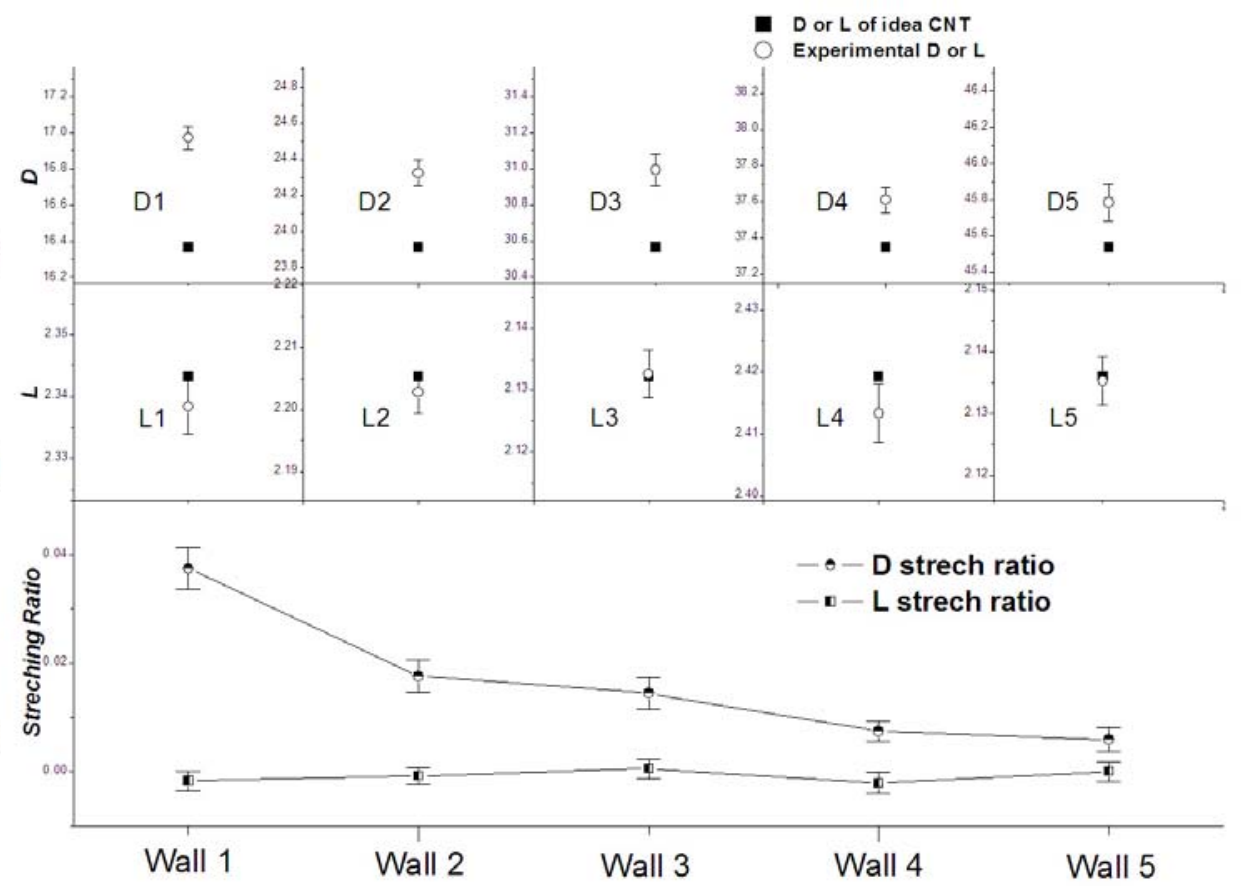

FIG. 2. Experimental diameter $D$ and axial periodicity $L$ are compared with the value of idea CNT of grapheme model in (a) and (b), respectively. The radial and axial stretching ratios are plotted in (c), and the diameter dependence of radial stretching is clear. 